\title{
Neuropsychological Outcome After Temporal Lobe Epilepsy Surgery in Patients With Postoperative Seizures following Unsuccessful Surgical Therapy
}

\author{
Abuhuziefa Abubakr ${ }^{\mathrm{a}, \mathrm{c}}$, Olukayode Onasanya ${ }^{\mathrm{b}}$
}

\begin{abstract}
Background: About $20-30 \%$ of patients with intractable partial epilepsy have less than favorable outcome after temporal lobectomy. This study explored the neuropsychological outcome of such surgery in this group of patients.
\end{abstract}

Methods: The Medical records of patients with intractable temporal lobe epilepsy who underwent temporal lobectomy were reviewed. All patients had detailed phase I presurgical evaluation and subsequently had surgery. Detailed neuropsychology assessment was carried out in all patients pre-operatively as well as between 3 and 12 months following surgery, 13 patients with Engel class 3 outcome were included in the study.

Results: There was no significant difference in the pre and post surgical IQ, verbal and visual-spatial memory performance. However, $76.9 \%$ of the patients improved in attention/executive function, $62.6 \%$ improved in language function and $88.6 \%$ improved in psycho-emotional function.

Conclusions: Despite suboptimal epilepsy surgery outcome, this study demonstrates that attention /executive function, language, and psycho-emotional function improved substantially in this sample of patients.

Keywords: Neuropsychology; Poor sezure outcome; Cognition; Language; Memory; Epilepsy surgery; Psychoemotion and inelligence

Manuscript accepted for publication October 1, 2012

${ }^{a}$ University of Mississippi Medical Center, 2500 North state street, Jackson MS 39216, USA

bJKK Hospital, 65 James Street, Edison NJ- 08818, USA

${ }^{\mathrm{c}}$ Corresponding author: Abuhuziefa Abubak, University of Mississippi

Medical Center, 2500 North state street, Jackson MS 39216, USA.

Email: huzy4tongor@gmail.com

doi: http://dx.doi.org/10.4021/jnr149w

\section{Introduction}

Approximately $80 \%$ of focal epilepsies originate in the temporal lobes [1]. Temporal lobe epilepsy surgery offers an effective therapeutic option for intractable, pharmacoresistant epilepsy, with $70-80 \%$ of patients demonstrating favorable seizure outcome (Engel class 1) after surgery [2-6]. Most studies have consistently demonstrated improvements in various neuropsychological domains such as verbal and executive functions in this group of patients following surgery [7-10]. On the other hand, it has been consistently reported that verbal memory is likely to decrease after resection of the language-dominant temporal lobe $[6,11,12]$. Identified risk factors for post operative cognitive decline after left temporal lobectomy are onset of seizures at later age, male gender, and a good preoperative cognitive/memory function [3]. A tendency to a decline in the visual spatial (non-verbal) memory has been found to exist after a right temporal lobectomy as well $[3,13]$.

However there is very little information reported about the neuropsychological outcome in the $20-30 \%$ of patients who continue to have seizures post operatively. Therefore in this study, we assessed the neuropsychological outcome of consecutive patients who had temporal lobectomy for refractory epilepsy and continued to have recurrent seizures, Engel's class 3 outcomes (worthwhile improvement $>50 \%$ reduction in seizures) [14].

\section{Methods}

We identified all patients with intractable temporal lobe epilepsy (performed retrospective review of all the medical records of patients with intractable temporal lobe epilepsy) who underwent temporal lobectomy between 1999 and 2007 at JFK hospital and performed retrospective review of all their medical records. All patients had a detailed phase I presurgical evaluation. This included continuous video-EEG recordings obtained through electrodes applied to the scalp according to 10 - 20 international system plus additional spheniodal electrodes, as well as detailed neuroimaging in- 
Table1. Clinical Data of IQ Analysis of Both Left and Right Temporal Lobes

\begin{tabular}{lllll}
\hline $\mathbf{N = 1 3}$ & Lobectomy Site & Improved & Unchanged & Worse \\
\hline Total IQ & Left Temporal & $38.5 \%(5)$ & 0 & $30.7 \%(4)$ \\
& Right Temporal & $15.4 \%(2)$ & 0 & $15.4 \%(2)$ \\
Verbal IQ & Left Temporal & $23 \%(3)$ & 0 & $46.2 \%(6)$ \\
& Right Temporal & $15.4 \%(2)$ & $1(7.7 \%)$ & $7.7 \%(1)$ \\
Performance IQ & Left Temporal & $38.4 \%(5)$ & 0 & $30.7 \%(4)$ \\
& Right Temporal & $7.7 \%(1)$ & $1(7.7 \%)$ & $15.4 \%(2)$ \\
\hline
\end{tabular}

cluding MRI, FDG-PET scan and Wada tests were obtained. All patients had standard en-bloc resection of temporal lobe and patients with Engel's class 3 outcomes and continued the same AEDs during the assessment period were included in the analysis.

Detailed neuropsychological assessment was carried out on all the patients preoperatively as well as at 3 months and 12 months postoperatively. Various test batteries were employed to assess neuropsychological domains that included overall Intelligent Quotient (IQ), Attention/Executive function, Language function, Visual-spatial memory, verbal memory and psycho-emotional function. The functional level of each respective domain of the neuropsychological tests at three and 12 months post surgery were compared with the preoperative level and the summary of the evaluation is classified as improved, unchanged or worse. For the purpose of analysis improved and unchanged cases were added together and compared to worsened patients.

\section{Neuropsychological battery}

The various tests were grouped and assessed as follows.

\section{Psychometric intelligence}

The WAIS-R was examined as a function of traditional threefactor solution; verbal comprehension, perceptual organiza- tion, and freedom from distraction [15].

\section{Language}

The visual naming and aural comprehension subsets of the Multilingual Aphasia Examination were used for analysis. The former provides a measure of expressive semanticretrieval-based language ability, while the latter provides a measure of receptive language comprehension ability.

\section{Attention/executive function}

Wisconsin Card sorting Test (WCST; Heaton, 1981) was selected to assess the higher order task solving, abstraction, and cognitive flexibility.

\section{Visual-spatial memory function}

Facial Recognition Test (Benson, Hamsher, Varney, and Spreen, 1983) was employed to assess the ability to recognize and discriminate among unfamiliar faces while Judgment of Line orientation Test (Benton, 1983) was employed to assess patients' spatial orientation adequacy.

\section{Memory}

Two indices were selected from the California Verbal Learn-

Table2. Total Results: Summary of IQ Analysis

\begin{tabular}{llll}
\hline & Improved & Unchanged & Worse \\
\cline { 2 - 3 } Total IQ & $54.3 \%(7)$ & 0 & $46.7 \%(6)$ \\
Verbal IQ & $38.8 \%(5)$ & $1(7.7 \%)$ & $53.9 \%(7)$ \\
Performance IQ & $46.15 \%(6)$ & $1(7.7 \%)$ & $46.15 \%(6)$
\end{tabular}


Table3. Clinical Data and Analysis of Memory and Attention of Left and Right Temporal Lobes

\begin{tabular}{lllll}
\hline $\mathbf{N}=\mathbf{1 3}$ & Lobectomy Site & Improved & Unchanged & Worse \\
\hline Attention/Executive function & Left Temporal & $46.7 \%(6)$ & 0 & $23 \%(3)$ \\
& Right Temporal & $23 \%(3)$ & $7.7 \%(1)$ & $(0)$ \\
Language & Left Temporal & $15.4 \%(2)$ & $30.7 \%(4)$ & $30.7 \%(4)$ \\
& Right Temporal & $23 \%(3)$ & $7.7 \%(1)$ & $23 \%(3)$ \\
Visuo-spatial memory & Left Temporal & $30.7 \%(4)$ & $15.4 \%(2)$ & $15.4 \%(2)$ \\
& Right Temporal & $15.4 \%(2)$ & 0 & $30.7 \%(4)$ \\
Verbal memory & Left Temporal & $30.7 \%(4)$ & $7.7 \%(1)$ & $15.4 \%(2)$ \\
& Right Temporal & $15.4 \%(2)$ & 0 & $15.4 \%(2)$ \\
\hline
\end{tabular}

ing Test (CVLT): total words recalled, and short-delay free recall; From the Wechsler Memory Scale we presented the Associate Learning, Logical Memory (delayed), and Visual Reproduction (delayed) subsets [15].

\section{Results}

We identified eighteen consecutive patients with Engel Class 3 outcome in the medical records of patients with intractable temporal lobe epilepsy (TLE) who underwent temporal lobectomy. Five out of the eighteen patients were excluded due to incomplete information. Therefore 13 patients were included in the analysis. Their age ranged from 19 to 52 years. Four had right temporal lobectomy and nine had left tem- poral lobectomy. The histopathology in 5 patients showed mesial temporal sclerosis and concordant with MRI of the brain except one where the MRI was reported as normal. Six patients had normal histopathology, and one patient's had microdysgenesis and another patient showed gliosis with ectopic neurons in the white matter. All the eight patients had normal MRI of the brain. All 13 patients were on the same preoperative AEDs during the assessment period following surgery, the overall range of AEDs were 2 to 3 drugs since none of the patients were rendered seizure free.

The data showed that the total IQ, verbal and performance IQ improved in 50\% of the subjects which was not significantly different from patients with worsened IQ level (Table 1,2). Similarly for verbal and visual-spatial memory, there was no substantial difference between the numbers of

Table4. Summary of Attention and Memory Analysis

\begin{tabular}{llll}
\hline & Improved & Unchanged & Worse \\
\cline { 1 - 2 } Attention/Executive Function & $69.2 \%(9)$ & $7.7 \%(1)$ & $23.1 \%(3)$ \\
Language & $38.4 \%(5)$ & $30.8 \%(4)$ & $30.8 \%(4)$ \\
Visuo-Spatial Memory & $46.2 \%(6)$ & $15.4 \%(2)$ & $38.4 \%(5)$ \\
Verbal memory & $46.15 \%(6)$ & $7.7 \%(1)$ & $46.15 \%(6)$ \\
Psycho-Emotional Function & $65.5 \%(8)$ & $23.1 \%(3)$ & $15.4 \%(2)$ \\
\hline
\end{tabular}


Table5. Over all Summaries of Patients With Improved/Unchanged and Worsened Scores on Several Neuropsychological Functions

\begin{tabular}{lll}
\hline & Improved/Unchanged & Worse \\
\hline Attention/Executive Function & $76.9 \%$ & $23.1 \%$ \\
Language & $69.2 \%$ & $30.8 \%$ \\
Visual-Spatial Memory & $61.6 \%$ & $38.4 \%$ \\
Verbal memory & $53.85 \%$ & $46.15 \%$ \\
Psycho-Emotional Function & $88.6 \%$ & $15.4 \%$ \\
\hline
\end{tabular}

patient that improved/remained unchanged versus those that worsened (Table 3, 4). However, a substantial number of patients improved on attention/executive function, language and psycho-emotional function as shown in Table 5 below.

\section{Discussion}

This study demonstrates selective improvements in cognitive performance in patients who had temporal lobectomy with poor surgical outcome and who continued to have seizures. Attention and executive function generally improved following surgery irrespective of the side of the surgery. Similarly language improvement was noted in the majority of our cohort since most of them (nine out of thirteen) had left temporal lobectomy. Helmstaedter et al [5] assessed the efficacy of short-term effects of cognitive rehabilitation on memory outcome of patients who had temporal lobectomy for refractory epilepsy. They compared a group of patients who received postoperative cognitive rehabilitation to a group of patients who did not. They found that rehabilitation improved verbal learning rather than memory. However, attention improved irrespective of whether patients received rehabilitation or not. It is of note that Helmstaedter et al only assessed seizure-free patients. This is in contrast to our study in which all our patients continued to have seizures following surgery. Moreover, our subjects did not undergo any constructive rehabilitation program. Elger et al [16] compared cognition and memory functions preoperatively and at 2 to 10 years postoperatively in medically versus surgically treated patients with refractory epilepsy. They found that memory and cognitive functions were better in seizure-free patients compared to patients who were treated medically. However, there was no difference between patients who had left temporal lobectomy and patients who continued to have seizures postoperatively. In contrast to our study, Elger et al's results may be influenced by the seizure free patients and the prolonged longitudinal nature of their study. In our study, we assessed our patients within a year following surgery. Furthermore, none of our patients were seizure free. Overall, our study showed that cognitive decline can be halted and even reversed by surgery. Attention has been found to improve after temporal lobectomy, regardless of the side of surgery [3]. Our study showed substantial improvement in attention/ executive function at 3 and 12 months post operatively in the majority of our patients. This is in accord with the Sawrie et al [17] study, in which they administered a series of cognitive and health related quality of life measures at baseline and 12 months after temporal lobectomy in 65 patients with TLE. They found that postoperative emotional distress predicted self-reported memory problems in addition to postoperative medication side effects and seizure freedom. They concluded that subjective and objective memory changes after temporal lobectomy are not related, however in contrast to their study our finding only in patients who failed surgery and continued to have seizures. Similarly our study demonstrates substantial improvement in language and psycho-emotional functions in $69.2 \%$ and $88.6 \%$ patients respectively.

Pharmacotherapy fails to control seizures in approximately $30 \%$ of patients. Temporal lobe surgery has been proved beneficial and effective for seizure control in this population of patients. The after effect of surgery has been a subject of controversy [3]. Most studies revealed some worsening of the memory function after temporal lobectomy in the language dominant hemisphere. The vast majority of these studies focused on patients who at least benefitted from surgery in seizure outcome (Engel class 1 and 2). The goal of our study was to evaluate whether improvements existed in the cognitive functions of patients who failed surgical therapy. The relative stability of IQ measures in this study population may indicate that general intellectual ability is not affected by temporal lobe resection [18]. Holmes et al [19] suggest that intractable temporal lobe epilepsy may cause a progressive deterioration in the higher cortical attention/executive and language functions. Psycho-emotional function also deteriorates over time since the limbic system 
is closely associated with the temporal lobes. Therefore it is not surprising that resection of such epileptogenic temporal lobe focus may arrest the deterioration and even bring about improvement in these neuropsychological domains as exemplified by the study.

Several studies evaluated the predictors of long-term seizure outcome after epilepsy surgery and there are conflicting results; Jutila et al [20] evaluated the long-term outcome in 140 consecutive patients who undergone temporal lobectomy for epilepsy and found that over half had been seizure free over a median follow-up of 2.6 years. They concluded that the best predictor for seizure freedom one year after surgery is unilateral MRI imaging abnormalities and early onset of seizures. This is in contrast to the study by Salanova et al, where they performed a longitudinal follow-up of 145 patients with TLE who underwent temporal lobectomy. They found that $66 \%$ were seizure free at 1 year, $60 \%$ at 5 years and $55 \%$ at 10 years follow-up and concluded that most of their patients with late recurrence of seizures had mesial temporal sclerosis. Compared to Julita et al study, Salanova et al [21] patients had a very long follow-up period which may explain the discrepancy in their conclusion. In our study most of the patients had a normal MRI and histopathology report. Cohen-Gadol et al [22] assessed 12 consecutive patients with mesial temporal lobe epilepsy and normal MRI and compared them to 50 patients with MTS. They coined the term paradoxical temporal lobe epilepsy (PTLE) for those patients with normal MRI. During the follow-up period $50 \%$ of PTLE patients were seizure free compared to $76 \%$ of the MTS. They performed further electrophysiological tests of the hippocampal tissues and demonstrate that dentate granule cells are less excitable in patients with PTLE compared to MTS group and they concluded that in MTS the damaged hippocampus is primary focus for the epileptogenisis, however in PTLE, extrahippocampal limbic structure may be the primary focus and secondarily involved the hippocampus, and by removing only the medial structure during anterior temporal lobectomy may not be sufficient to render the patients with PTLE seizure free. Their clinical findings were somewhat similar to ours such that the majority of the patients in our cohort had a normal MRI and pathology report, of course in our study we did not assessed the electrophysiology of the surgical specimens, nor the re-operation assessment.

The limitation of our study is that it's a retrospective review of data and there is no controlled arm. Additional limitation includes lack of quality of life assessment. Future studies might examine these measures at a longer follow-up interval in a prospective cohort.

In conclusion, this study demonstrated that in-spite of suboptimal seizure outcome following temporal lobe surgery; there was a substantial improvement in attention and executive function, language and psycho-emotional functions in the current study sample. These results may be in- cluded to counsel patients about cognitive improvement in a setting of incomplete resolution of seizures following surgery.

\section{References}

1. Spencer SS, Berg AT, Vickrey BG, Sperling MR, Bazil CW, Shinnar S, Langfitt JT, et al. Initial outcomes in the Multicenter Study of Epilepsy Surgery. Neurology. 2003;61(12):1680-1685.

2. Yoon HH, Kwon HL, Mattson RH, Spencer DD, Spencer SS. Long-term seizure outcome in patients initially seizure-free after resective epilepsy surgery. Neurology. 2003;61(4):445-450.

3. Martin RC, Sawrie SM, Roth DL, Gilliam FG, Faught E, Morawetz RB, Kuzniecky R. Individual memory change after anterior temporal lobectomy: a base rate analysis using regression-based outcome methodology. Epilepsia. 1998;39(10):1075-1082.

4. York MK, Rettig GM, Grossman RG, Hamilton WJ, Armstrong DD, Levin HS, Mizrahi EM. Seizure control and cognitive outcome after temporal lobectomy: a comparison of classic Ammon's horn sclerosis, atypical mesial temporal sclerosis, and tumoral pathologies. Epilepsia. 2003;44(3):387-398.

5. Helmstaedter C, Loer B, Wohlfahrt R, Hammen A, Saar J, Steinhoff BJ, Quiske A, et al. The effects of cognitive rehabilitation on memory outcome after temporal lobe epilepsy surgery. Epilepsy Behav. 2008;12(3):402-409.

6. Rausch R, Kraemer S, Pietras CJ, Le M, Vickrey BG, Passaro EA. Early and late cognitive changes following temporal lobe surgery for epilepsy. Neurology. 2003;60(6):951-959.

7. Chelune GJ, Naugle RI, Luders H, Awad IA. Prediction of cognitive change as a function of preoperative ability status among temporal lobectomy patients seen at 6-month follow-up. Neurology. 1991;41(3):399-404.

8. Alpherts WC, Vermeulen J, Hendriks MP, Franken ML, van Rijen PC, Lopes da Silva FH, van Veelen CW. Long-term effects of temporal lobectomy on intelligence. Neurology. 2004;62(4):607-611.

9. Gleissner U, Helmstaedter C, Schramm J, Elger CE. Memory outcome after selective amygdalohippocampectomy in patients with temporal lobe epilepsy: oneyear follow-up. Epilepsia. 2004;45(8):960-962.

10. Lendt M, Helmstaedter C, Elger CE. Pre- and postoperative neuropsychological profiles in children and adolescents with temporal lobe epilepsy. Epilepsia. 1999;40(11):1543-1550.

11. Alpherts WC, Vermeulen J, van Rijen PC, da Silva FH, van Veelen $\mathrm{CW}$. Verbal memory decline after temporal epilepsy surgery?: A 6-year multiple assessments follow-up study. Neurology. 2006;67(4):626-631. 
12. Bjornaes H, Stabell KE, Roste GK, Bakke SJ. Changes in verbal and nonverbal memory following anterior temporal lobe surgery for refractory seizures: effects of sex and laterality. Epilepsy Behav. 2005;6(1):71-84.

13. Morris RG, Abrahams S, Polkey CE. Recognition memory for words and faces following unilateral temporal lobectomy. Br J Clin Psychol. 1995;34 ( Pt 4)(571-576.

14. Engel J Jr, Van Ness PC, Rasmussen TB, Ojemann LM. Outcome with respect to epileptic seizures; in Engel J Jr (ed): Surgical treatment of epilepsy, 2nd ed. NY, Raven press 1993:609-621.

15. Seidenberg M, Hermann B, Wyler AR, Davies K, Dohan FC, Jr., Leveroni C. Neuropsychological outcome following anterior temporal lobectomy in patients with and without the syndrome of mesial temporal lobe epilepsy. Neuropsychology. 1998;12(2):303-316.

16. Helmstaedter C, Kurthen M, Lux S, Reuber M, Elger CE. Chronic epilepsy and cognition: a longitudinal study in temporal lobe epilepsy. Ann Neurol. 2003;54(4):425432.

17. Sawrie SM, Martin RC, Kuzniecky R, Faught E, Morawetz R, Jamil F, Viikinsalo M, et al. Subjective versus objective memory change after temporal lobe epilepsy surgery. Neurology. 1999;53(7):1511-1517.

18. Szabo CA, Wyllie E, Stanford LD, Geckler C, Kotagal P, Comair YG, Thornton AE. Neuropsychological effect of temporal lobe resection in preadolescent children with epilepsy. Epilepsia. 1998;39(8):814-819.

19. Holmes MD, Dodrill CB, Wilkus RJ, Ojemann LM, Ojemann GA. Is partial epilepsy progressive? Ten-year follow-up of EEG and neuropsychological changes in adults with partial seizures. Epilepsia. 1998;39(11):1189-1193.

20. Jutila L, Immonen A, Mervaala E, Partanen J, Partanen $\mathrm{K}$, Puranen M, Kalviainen R, et al. Long term outcome of temporal lobe epilepsy surgery: analyses of 140 consecutive patients. J Neurol Neurosurg Psychiatry. 2002;73(5):486-494.

21. Salanova V, Markand O, Worth R. Longitudinal followup in 145 patients with medically refractory temporal lobe epilepsy treated surgically between 1984 and 1995. Epilepsia. 1999;40(10):1417-1423.

22. Cohen-Gadol AA, Bradley CC, Williamson A, Kim JH, Westerveld M, Duckrow RB, Spencer DD. Normal magnetic resonance imaging and medial temporal lobe epilepsy: the clinical syndrome of paradoxical temporal lobe epilepsy. J Neurosurg. 2005;102(5):902-909. 